\title{
A Hybrid Structural Health Monitoring System for the Detection and Localization of Damage in Composite Structures
}

\author{
Darun Barazanchy, ${ }^{1}$ Marcias Martinez, ${ }^{1}$ Bruno Rocha, ${ }^{2}$ and Marko Yanishevsky ${ }^{2}$ \\ ${ }^{1}$ Faculty of Aerospace Engineering, Delft University of Technology, Kluyverweg 1, P.O. Box 5058, 2600 GB Delft, The Netherlands \\ ${ }^{2}$ Structures, Materials and Manufacturing, National Research Council Canada (NRC), 1200 Montreal Road, \\ Ottawa, ON, Canada K1A 0R6 \\ Correspondence should be addressed to Marcias Martinez; m.j.martinez@tudelft.nl
}

Received 11 June 2014; Revised 15 September 2014; Accepted 28 September 2014; Published 29 October 2014

Academic Editor: Yanbiao Liao

Copyright (C) 2014 Darun Barazanchy et al. This is an open access article distributed under the Creative Commons Attribution License, which permits unrestricted use, distribution, and reproduction in any medium, provided the original work is properly cited.

\begin{abstract}
A hybrid structural health monitoring (SHM) system, consisting of a piezoelectric transducer and fiber optic sensors (FOS) for generating and monitoring Lamb waves, was investigated to determine their potential for damage detection and localization in composite aerospace structures. As part of this study, the proposed hybrid SHM system, together with an in-house developed algorithm, was evaluated to detect and localize two types of damage: a through thickness damage (hole of $2 \mathrm{~mm}$ in diameter) and a surface damage $(2 \mathrm{~mm}$ diameter bore hole with a depth of $0.65 \mathrm{~mm}$ ) located on the backside of the plate. The experiments were performed using an aircraft representative composite plate skin, manufactured from carbon fiber reinforced polymer (CFRP).
\end{abstract}

\section{Introduction}

In accordance with current regulations and requirements, an aircraft's structural integrity is ensured using standard nondestructive evaluation (NDE) techniques such as eddycurrent, liquid penetrant, and ultrasonics to name a few [1]. Unfortunately, the application of these NDE techniques tends to require an aircraft to be taken out of service for substantial periods of time, resulting in considerable costs (downtime costs and maintenance costs) for the operator. To reduce costs, original equipment manufacturers (OEM), operators, maintainers, and researchers have been envisioning systems which can be integrated into the structure making NDE an integral part of the structure. This approach would provide an operator with the capability to inspect the aircraft before or after each flight, or during the flight itself. The aircraft would only need to be taken out of service when damage is actually detected and requires maintenance/repair action, rather than after a certain defined period of time, or number of flights. The research field that aims at developing such systems and the methods of data acquisition and analysis to evaluate the health of a structure is known as structural health monitoring (SHM) $[2,3]$.
Within the field of SHM several techniques, sensors, and systems have been devised, developed, and demonstrated/tested, which can be used to evaluate the condition of a structure. One of these proposed techniques uses fiber optic sensors (FOS) for damage detection based on guided ultrasonic waves (GUW) [4-7]. Guided ultrasonic waves, as described by Lamb [8], have many favorable attributes such as their small amplitude attenuation over long distances $[4,5]$. Optical fibers have been the subject of considerable development, having been used extensively for data communication, and exhibit excellent fatigue characteristics [9]. In addition, FOS are lightweight and small in dimension, are immune to electromagnetic interference, and have good resistance to chemicals and the environment. Furthermore, they have already been successfully integrated, either attached to the surface of or embedded into composite laminate plates $[2,10]$.

For the application of FOS in SHM, a light source and light detection system are needed to illuminate and interrogate the sensor(s), respectively. Multiple interrogation systems have been proposed to measure strains; however, in the case of Lamb waves and related strains, the required frequency for data acquisition introduces stringent requirements regarding the interrogation system and limits the 
number of sensors that can be integrated into such interrogation systems. The FOS based systems which can be used are based on point sensing interferometric techniques (e.g., fiber Bragg grating (FBG) based, or Fabry-Perot (FP) based [11]). Fabry-Perot interferometric techniques present, however, added difficulties in terms of sensor multiplexing in the same optical fiber when compared with FBGs.

A downside of fiber optics is that they can only be used as sensors, or in actuating systems involving high power lasers, resulting in complex in-the-field applications. Therefore, there will always be a need for some sort of simple actuation mechanism to generate the Lamb wave, which can be effectively achieved using a piezoceramic transducer. Fortunately, piezoceramic transducers scanning Lamb waves, which can be used both as sensors and actuators, are inexpensive and available in small sizes making them ideal for integration into structures [12]. Unfortunately, piezoceramic transducers and related electrical wiring for power and/or data acquisition can be affected by and/or be the source of electromagnetic interference impacting the performance of other avionic systems on an aircraft. These undesirable effects of piezoceramic transducers need to be considered and evaluated prior to their use in in-service applications. Qiu et al. [13], demonstrated that proper shielding of these piezoceramic transducers can reduce these undesirable effects.

Takeda et al. [14] presented a hybrid SHM system based on Lamb waves which detected damage in composite structures. The proposed hybrid SHM system used a piezoceramic transducer to generate Lamb waves and FBGs to detect them. In this interrogation architecture, a broadband light source (BBS) was used to illuminate the entire FBG sensors spectra on an arrayed waveguide grating (AWG) detection system. When the FBG spectrum shifted due to the waves' strain field, the light power output of the sensitive channels of the AWG also varied. The optical output of each channel was converted to an electrical (voltage) signal using a photodetector, which was captured by a high sampling frequency data acquisition system sensing the propagating Lamb waves. This propagated signal included excited waves produced by a piezoceramic transducer and those generated from damage reflections resulting from the interference of damage with the original actuated and propagating Lamb wave field. These reflections were detected through the comparison of signals obtained from the same sensor for different conditions of the structure being inspected, that is, undamaged baseline signal and damaged state signal. Having FBG sensors with different orientations and in different locations of the structure enabled the detection and the localization of damage through the use of time of flight (ToF) and propagation velocity and calculated traveled distances of the waves by the application of triangulation algorithms and/or by considering the directional sensitiveness of the FBGs. These sensors were sensitive to strains applied along their length and therefore, different FBGs with different orientations were able to sense a propagating wave and output different signal amplitudes. The comparison of these amplitudes enabled the determination of the incoming direction of the wave.

Regarding the selection of the FBG sensors, Takeda et al. [14] reported the ideal length of an FBG sensor for wave detection to be $1 / 7$ of the source wavelength. This relation reflects a compromise between desired point sensing (as opposed to averaging strains along the sensor) and reflectivity of the FBG (related with grating length), which is essential to attain a sufficiently high signal to noise ratio (SNR), besides avoiding nonlinearity effects in the strain sensing.

Takeda et al. [14] evaluated their SHM system using an artificially introduced delamination in a small composite plate ( 240 by 240 by $1 \mathrm{~mm}$ ) between a piezoceramic transducer and FBG located $50 \mathrm{~mm}$ apart. In addition, they reported the threshold for a detectable delamination size to be $10 \mathrm{~mm}$ in width. The detection was based on a new wave (accelerated mode) detected between the $S_{0}$ and $A_{0}$ wave, which was not present in the original signal for the undamaged plate. The accelerated mode was considered to be caused by the separation of $A_{0}$ wave into two propagation paths and conversion of the original $A_{0}$ wave into an $S_{0}$ and $A_{0}$ wave along the different paths [14].

Tsuda [15] and Lam et al. [16] presented an interrogation architecture based on a secondary FBG filter cascaded with the FBG sensor. This architecture used a BBS with two FBGs. The first FBG caused a phase shift in reflected spectrum, which intersected with the spectrum of the second FBG sensor. However, this architecture required the center wavelength of the FBGs to be within a close range of each other to operate successfully. Similar to the previously mentioned interrogation architecture, a photodetector and a data acquisition system were also used. Tsuda [15] detected, using the cascaded FBG interrogation architecture, a damage area of $65 \mathrm{~mm}$ in length and $15 \mathrm{~mm}$ in width. The studies of Lam et al. [16] reported a detected delamination of $20 \mathrm{~mm}$ in width between a piezoceramic transducer and FBG located $100 \mathrm{~mm}$ apart in a composite beam (440 by 25 by $2 \mathrm{~mm}$ ).

$\mathrm{Wu}$ et al. [17] successfully detected and localized damage in a composite laminate ( 305 by $152 \mathrm{~mm}$ ) using a hybrid pitchcatch SHM system. The damage, introduced by repeated impacts with a hammer, was located in an area (102 by $67 \mathrm{~mm}$ ) enclosed by two piezoceramic transducers and three FBGs. A damage index (DI), which represented the ratio of energy scatter between the damaged state and the undamaged baseline within a selected time window, was defined to predict the damage location. The diagnostic image obtained by Wu et al. [17] predicted a debond area of 30 by $30 \mathrm{~mm}$; this result was verified by X-ray imaging of the composite laminate.

Recently, Tan et al. [18] reported damage detection and localization in an aluminum plate ( 300 by 300 by $2 \mathrm{~mm}$ ) based on a similar SHM architecture as Betz et al. [4]. The damage, however, was located in the enclosed area between the transducer and the FBGs, and the localization algorithm was based on ToF obtained from four different FBGs. An improvement on the detectable damage size threshold was realized: $6 \mathrm{~mm}$ in diameter as compared to $12 \mathrm{~mm}$ reported by Betz et al. [4].

The research described in this paper focused on detection and localization of representative damage in aircraft structures using an elliptical triangulation algorithm [18, 19]. A composite plate with approximate dimensions of 1000 by 400 by $5.5 \mathrm{~mm}$ was used to evaluate the capabilities of the hybrid SHM system to detect and localize two types of damage. The 
first damage was a through thickness hole with a diameter of $2 \mathrm{~mm}$; the second was a representation of a surface damage, simulated by a bore hole $2 \mathrm{~mm}$ diameter with a depth of $0.65 \mathrm{~mm}$. It is important to note that the surface damage was located on the backside of the plate with respect to the surface upon which the sensors were bonded. This scenario simulated the detection of a damage on the external surface (e.g., on the external surface of composite aircraft skin), which is exposed to the environment, using sensors bonded on the inner surface, which would be protected from such environment. To the best of the authors knowledge, a hybrid SHM system which is capable of detecting damage as small as $2 \mathrm{~mm}$ in diameter in a composite panel and external surface damage of the same size located on the opposite face with respect to the one where the sensors are bonded is not presented in the literature. Furthermore, in the previously referenced literature, damage detection is presented for relatively high density of transducers used when compared with a relatively small scanned area. Additionally, damages were introduced in the area enclosed by the transducers, or in the direct path of two or more transducers. In this study, damages were introduced cumulatively inside, but also outside the area enclosed by the used transducers, and not in the direct path of any transducer combination. This reflects a real life scenario where small damage can occur in relatively large areas, outside the areas enclosed by applied transducers and while trying to maintain a low transducer density for simplicity, enabling a realistic future in-service application.

In this assessment only the tunable laser interrogation system was investigated for damage detection and localization purposes. Rocha et al. [20] evaluated three interrogator architectures described previously for their capability to detect Lamb waves, accurately and repeatedly, and reported that the tunable laser interrogation system provided the best results.

\section{Experimental Setup}

The experiments were performed using a realistic, aircraft representative composite plate skin. A quasi-isotropic carbon fiber reinforced polymer (CFRP) skin with a layup of $\left[( \pm 45)_{4} /(0 / 90)_{4}\right]_{s}$ consisted of 16 plies of Hexcel HexPly F593 Epoxy Resin F3T584 Carbon Fabric pre-preg. The composite plate layup followed the design guidelines discussed by Kassapoglou [21]; for example, the $\pm 45^{\circ}$ plies were placed on the outside of the plate to improve the (impact) damage resistance properties of the composite plate. The composite plate with dimensions of approximately 1000 by 400 by $5.5 \mathrm{~mm}$ was designed and manufactured to serve as a replacement skin for the SHM Platform 2 [22] developed at the National Research Council Canada (NRC).

The proposed hybrid interrogation system was evaluated for its capability to detect material acoustic waves. Initially, the properties of the quasi-isotropic CFRP laminate were calculated and the dispersion behavior of Lamb waves in the composite skin was estimated. In this study, the damage detection system was based on the faster fundamental symmetric $\left(S_{0}\right.$, with a group velocity of $4800 \mathrm{~m} / \mathrm{s}$ being also verified experimentally in the used skin) mode, since the excited waves of this mode do not suffer interference from the slower propagating waves of the fundamental antisymmetric $\left(A_{0}\right.$, $2800 \mathrm{~m} / \mathrm{s}$ ) mode. For the $S_{0}$ mode, based on the estimated dispersion behavior, a frequency range between 50 and $400 \mathrm{kHz}$ was selected in order to avoid the generation of higher order modes than the fundamental ones, $S_{0}$ and $A_{0}$, thereby simplifying the analysis of the sensor signals. The selected frequency range assured that the propagation velocities of the $S_{0}$ and $A_{0}$ modes were considerably different; therefore the interference between excited waves of these modes was minimized. The propagation velocities for the $S_{0}$ mode across the frequency range were fairly constant, resulting in similar phase and group velocities, which subsequently decreased the effects of spectrum spreading in the generation of Lamb waves.

The Lamb wave based hybrid SHM system described in this paper consisted of multiple FBG sensors and a piezoceramic disc actuator with a diameter of $6.35 \mathrm{~mm}$, bonded at the center of the composite plate. Considering the estimated and experimentally verified wavelength and frequency relation (dispersion), and the diameter of the actuator, tuning the detection technique to the $S_{0}$ waves resulted in the selection of a frequency of $325 \mathrm{kHz}$. The propagation velocity of the $S_{0}$ waves at this frequency was $4800 \mathrm{~m} / \mathrm{s}$, which resulted in a wavelength of approximately $15 \mathrm{~mm}$, as shown in Figures 1(a) and 1(b), respectively. Tuning the system to the $S_{0}$ wave was achieved by selecting a wavelength which was approximately double the actuator diameter, while trying to match the actuator diameter to a multiple of the $A_{0}$ wavelength with the same frequency.

A Hann windowed five-peak sinusoid with the selected frequency of $325 \mathrm{kHz}$ was used to minimize scattering in the frequency spectrum of the excited waves, minimizing the propagation of waves with different velocities. A Physical Acoustics Corporation 1410 arbitrary waveform signal generator was used, enabling the actuation of the piezoceramic transducer with a maximum voltage of $35 \mathrm{~V}$, at 100 megasamples per second (MS/s). The generated signal was used to trigger a Tektronix TDS 5104 oscilloscope. The output data were acquired at $125 \mathrm{MS} / \mathrm{s}$ during intervals of $400 \mu \mathrm{s}$, enabling the complete scan of the composite plate.

The selection of the FBG sensors was based on the previously mentioned work done by Takeda et al. [14], which found that the ideal length of an FBG sensor for Lamb wave detection would be 1/7 of the wavelength. For the selected frequency (and subsequently the selected wavelength), the length of the FBG sensors was determined to be $2.15 \mathrm{~mm}$. Due to availability of sensors, however, $5 \mathrm{~mm}$ long FBGs were used. Three pairs of FBGs written in single mode optical fibers (SMF-28) were bonded to the surface of the composite plate, following the process described in [23]. The locations and orientations $(\Lambda)$ of the sensors are depicted in Figure 2; their corresponding wavelengths $(\lambda)$ and positions with respect to the lower left corner of the plate are presented in Table 1.

The proposed interrogator configuration was completed (see Figure 3) using an Agilent 81640B fine linewidth tunable laser, with a maximum output power of $3.2 \mathrm{~mW}$, and a Thorlabs PDA10CS photodetector which was connected to the oscilloscope. Prior to each actuation, the laser output was tuned to a desired wavelength in the spectrum of one 


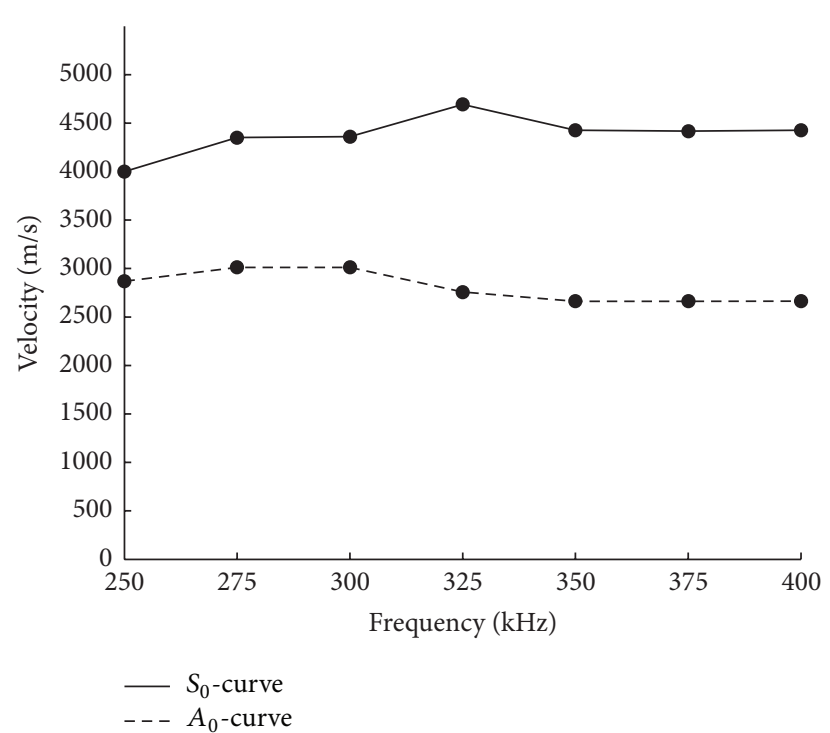

(a)

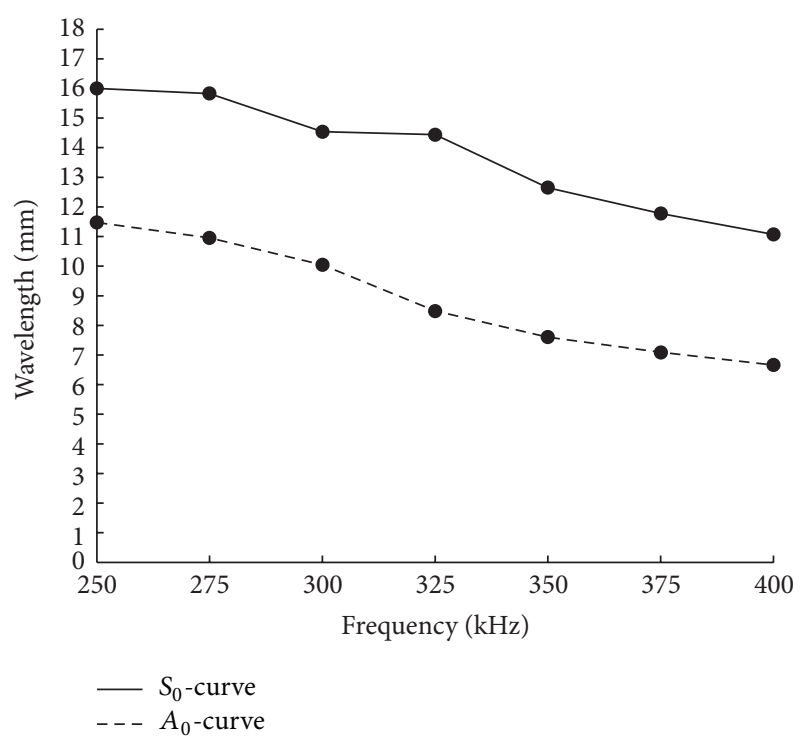

(b)

FIGURE 1: Experimentally obtained characteristics for the composite plate: (a) the dispersion curves and (b) the wavelength against actuation frequency curve.

TABLE 1: Wavelength $(\lambda)$ and position of the FBGs with respect to lower left corner of the plate and their orientation $(\Lambda)$.

\begin{tabular}{lcccc}
\hline FBG & $\lambda[\mathrm{mm}]$ & $x[\mathrm{~mm}]$ & $y[\mathrm{~mm}]$ & $\Lambda\left[^{\circ}\right]$ \\
\hline 1 & 1556.2 & 272.5 & 203.1 & 0 \\
2 & 1555.3 & 641.5 & 243.0 & 22.5 \\
3 & 1553.1 & 643.1 & 149.3 & 67.5 \\
4 & 1546.6 & 654.5 & 167.8 & 45 \\
5 & 1558.6 & 653.7 & 246.8 & 45 \\
6 & 1547.5 & 294.2 & 203.1 & 90 \\
\hline
\end{tabular}

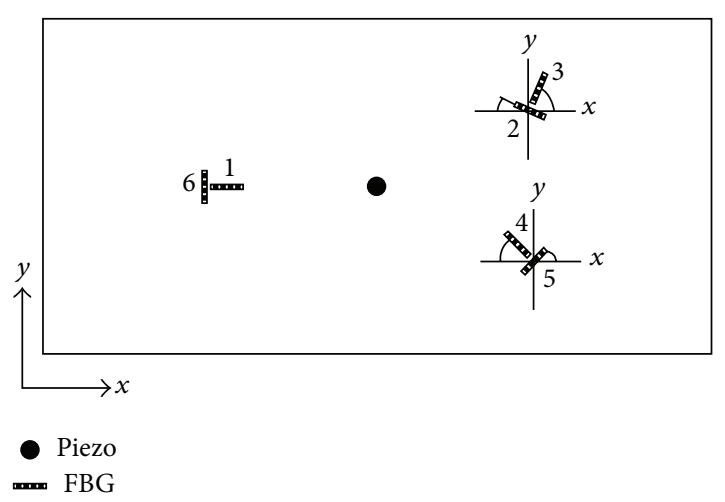

Figure 2: Schematic representation of the position of the FBG sensors on the composite plate.

FBG sensor, as explained previously, with this being repeated for all FBGs within one scan. With this interrogation architecture, the sensors were consistently capable of detecting the propagating Lamb waves with sufficient SNR. To reduce the effect of noise in the sensor data and to address the small SNR for the detection of Lamb waves' corresponding

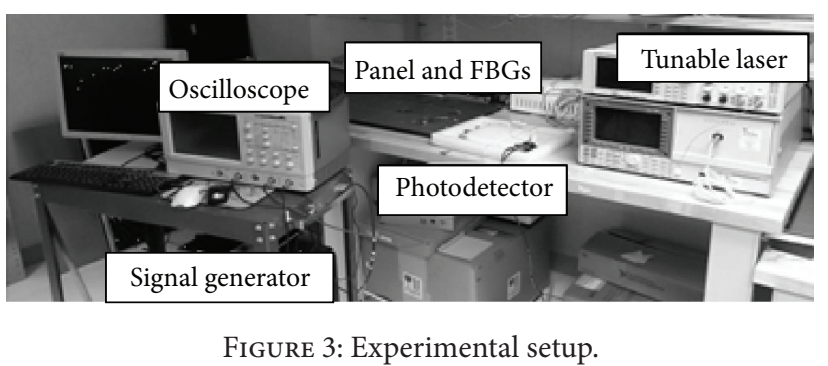

strain field sensor signal, multiple scans, that is, sequenced actuations, are performed repeatedly in a short period of time, for an approximate constant condition (health) of the structure under inspection. The data from these multiple tests are post-processed and condensed, through the formation of a signal band with outliers.

\section{Methodology and Results}

A damage detection and localization algorithm was developed and evaluated for the detection and localization of two different types of damage. In this section the damage detection and localization algorithm is introduced and elaborated first. Then, the evaluation of this algorithm for the two scenarios is presented: first for through thickness damage, then for surface damage, simulated by a bore hole, located on the backside of the plate with respect to the surface where the sensors were bonded.

3.1. Damage Detection and Localization Algorithm. For a pristine plate, baseline signals were acquired for all FBG sensors. To reduce the effects of noise in each scan, five signals were acquired for each FBG, corresponding to five actuation 


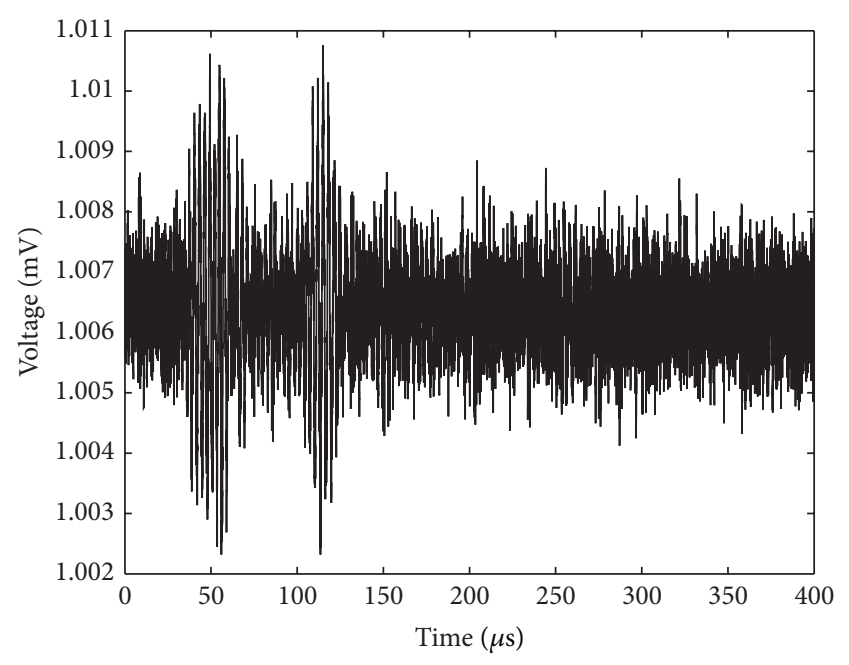

(a)

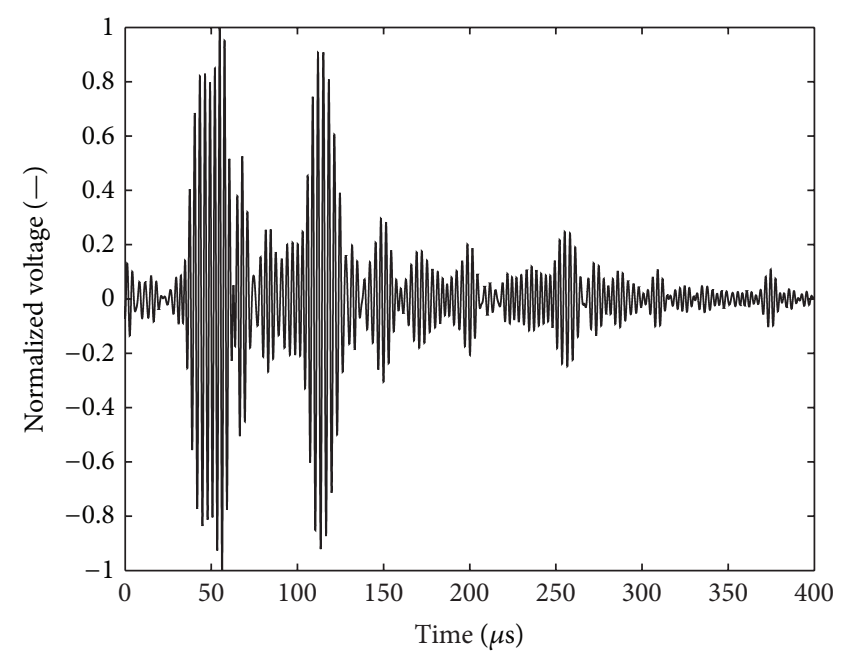

(b)

Figure 4: (a) Raw baseline signal of FBG 3 as obtained from the oscilloscope and (b) signal obtained after applying bandpass filter and normalization.

signals applied sequentially and therefore for approximately the same condition of the plate. The damage detection and localization algorithm filtered all raw signals with a Butterworth bandpass filter. This filter allowed signals between a frequency of $300 \mathrm{kHz}$ and $400 \mathrm{kHz}$ to be passed, while signals outside of this range were blocked. After the filter was applied the signals were normalized with respect to the highest amplitude occurring during the $400 \mu \mathrm{s}$ acquisition time period. Figure 4 shows one of the raw signals and its corresponding signal after the bandpass filter and normalization process.

After the introduction, or enlargement of each simulated damage, a scanning process similar to the one applied to obtain the baseline signals was implemented; that is, multiple actuations/scans were applied in sequence, with each FBG signal for each actuation being acquired, filtered, and normalized.

After the filtered and normalized baseline signals and damaged state corresponding signals were obtained, signalbands were formed for each FBG and each condition of the plate, considering for each time in the signals the maximum and minimum values obtained. The signal-bands obtained for each FBG for the damaged and baseline conditions were then compared. All the values of a newly obtained signal-band, the damaged state corresponding signal-band, which were within the signal-band filter obtained for baseline conditions, were set to zero, while the remaining signal-bands were kept and used in the damage localization algorithm. This was repeated for each condition of the plate tested, for each sensor and scan. This ensured that reflections due to the damage were repeatedly acquired, while signals present in both the states, for example, the originally actuated propagating waves corresponding signals, and noise, were discarded, or their effect was minimized. Furthermore, all possible differences obtained in this process, between the baseline and damage state corresponding signal-bands, were considered for the damage localization algorithm. A density of possible positive damage detection and localization was then obtained for each point in the plate - proportional to a probability of damage, or a damage index. For the application of the localization algorithm numerically the plate was meshed with a spacing of $5 \mathrm{~mm}$ in both planar directions. The effect of the application of the bandpass filter algorithm is shown in Figure 5, where a FBG signal is shown before and after the bandpass filtering was applied. In case there was no damage, all the values of the newly obtained signal were set to zero, and the algorithm for damage localization stopped.

The damage localization algorithm was based on the ToF (time of arrival with respect to the actuation signal of the highest peaks in the signals) after the signal-band filter was applied. From the experimentally determined dispersion curve (see Figure 1(a)), a range of propagation velocities for the $S_{0}$ wave was selected. The range, 4500 to $4800 \mathrm{~m} / \mathrm{s}$, was based on the $S_{0}$ wave velocity at a frequency of $325 \mathrm{kHz}$. With the $\mathrm{ToF}$ and propagation velocities, traveled distances were calculated. Each distance corresponds to the traveled distance of the excited $S_{0}$ wave between actuator and damage, plus the distance traveled by the damage reflection, between damage and the FBG considered, as shown in Figure 6(a). The total traveled distances, that is, the sum of the distances explained previously, for both velocities are constant and can be related to the characteristics of an ellipse (the sum of the distances between any point in an ellipse and its two focal points is constant). Therefore, for each actuator-sensor pair, all the possible damage locations are defined by an ellipse with focal points at the actuator and sensor locations $[18,19]$, as shown in Figure 6(b). When the process of ellipse construction was repeated for both velocities, a "ring" representing all possible damage locations was obtained for each actuatorsensor pair, as shown in Figure 6(c). The mathematics behind this procedure is given in

$$
\begin{aligned}
& x=x_{c}+a \cos (t) \cos (\phi)-b \sin (t) \sin (\phi) \\
& y=y_{c}+a \cos (t) \sin (\phi)+b \sin (t) \cos (\phi),
\end{aligned}
$$




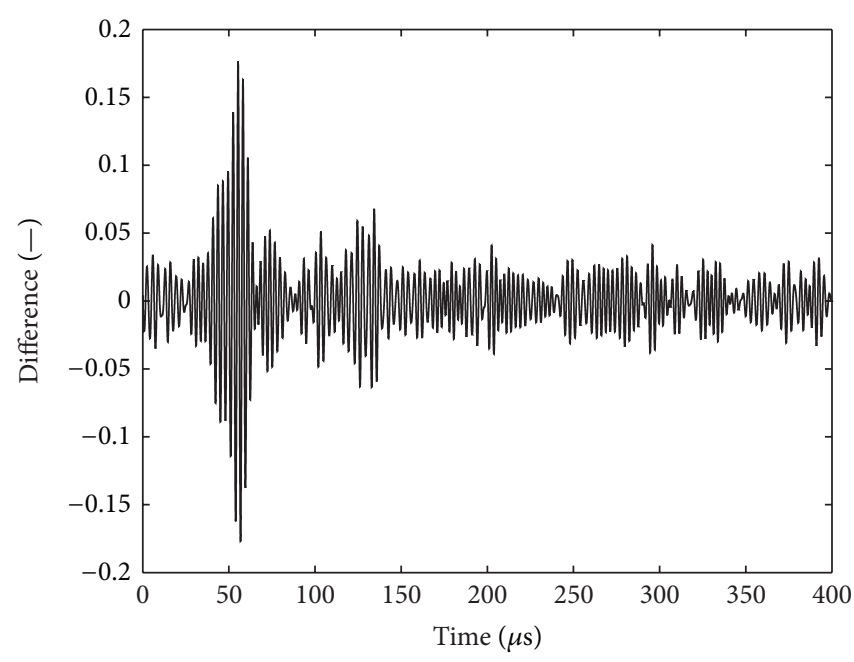

(a)

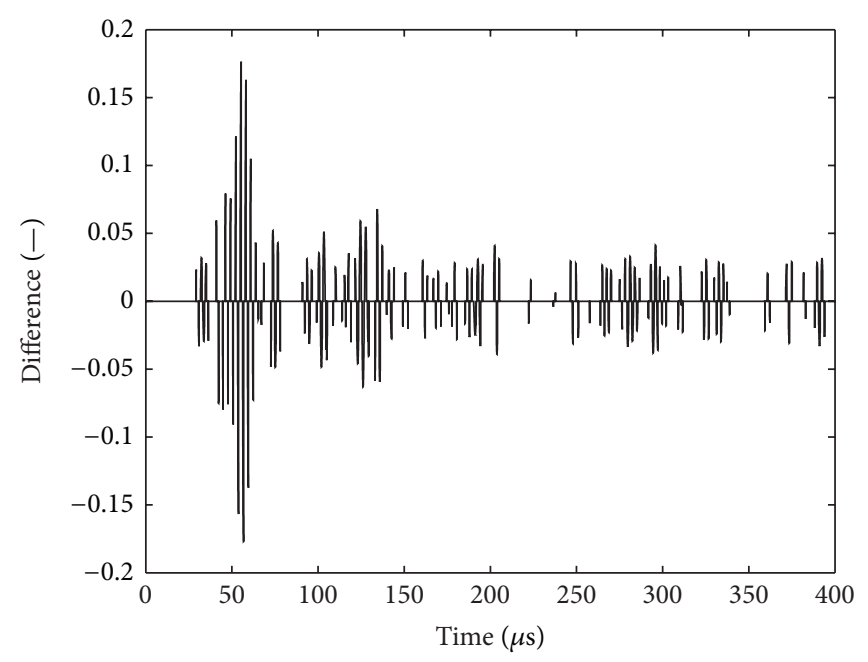

(b)

FIGURE 5: (a) Difference in signal of FBG 3 between baseline and damaged signal and (b) difference in signal of FBG 3 after the signal-band filter was applied.

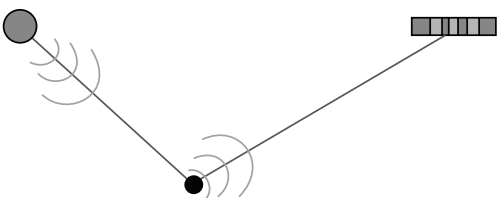

(a)

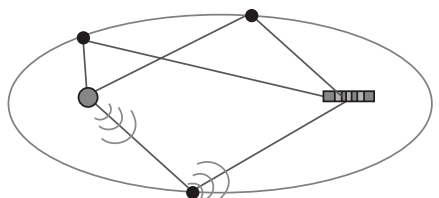

(b)

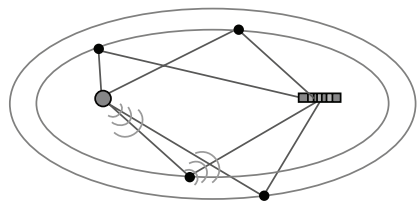

(c)

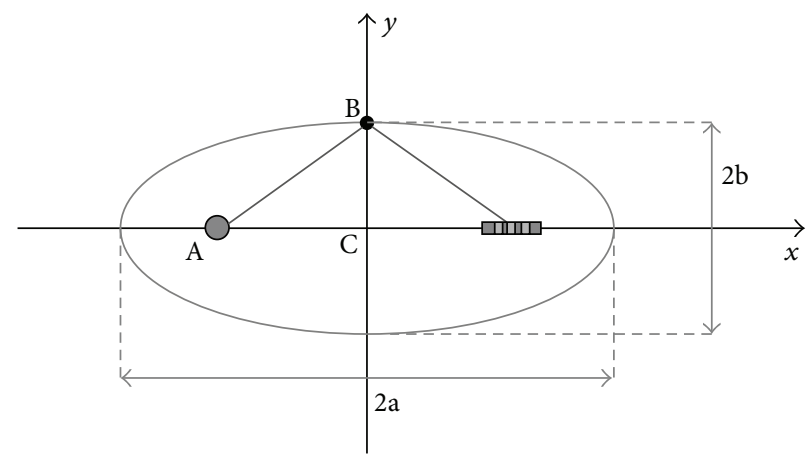

(d)

FIGURE 6: Illustration of constructing ellipses: (a) one possible location of damage, (b) all possible locations of damage, construction of one ellipse, (c) procedure repeated for both fixed velocities, construction of a ring for one combination of PZT and FBG, and (d) the important variables used.

where $x_{c}$ and $y_{c}$ are the $x$ - and $y$-coordinate of the center of the ellipse, and $\phi$ is the angle between the $x$-axis and the major axis of the ellipse; all three variable were calculated using the sensor locations, as shown in Table 1 . The parameter $t$ varied from 0 to $2 \pi$. The only unknown variable remained was the length of the minor axis, $2 \mathrm{~b}$, as shown in Figure 6(d). To calculate the length of the minor axis, the triangle $A B C$ was used, the line $\mathrm{AC}$ was equal to half the major axis, and the line $\mathrm{AB}$ was equal to half the distance between the sensors. The line $\mathrm{BC}$, which equals half of the minor axis, was calculated using Pythagoras theorem. This process was repeated for all the actuator-sensor pairs; the probable damage location on the plate would be associated with the area where most of the rings intersected.

It needs to be stated that the damage localization algorithm only investigated signals up to $100 \mu$ s after actuation. This limited the area which could be inspected by the sensor configuration used in this research. To obtain the maximum area which could be inspected using this sensor configuration, the time of arrival for each FBG was set to $100 \mu \mathrm{s}$, and the conservative lower wave propagation velocity was considered. These settings produced the extreme locations at which damage could be present. The algorithm, however, analyzes all times up to that maximum time interval; this 


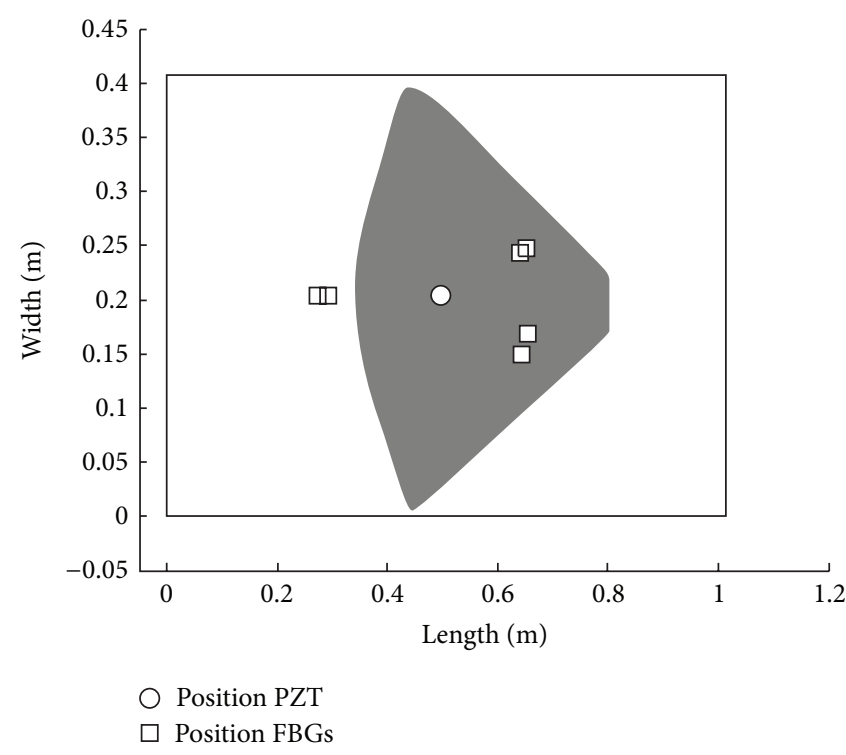

FIGURE 7: Plate area which can be inspected using the presented sensor configuration and analyzing signals up to $100 \mu$ s duration.

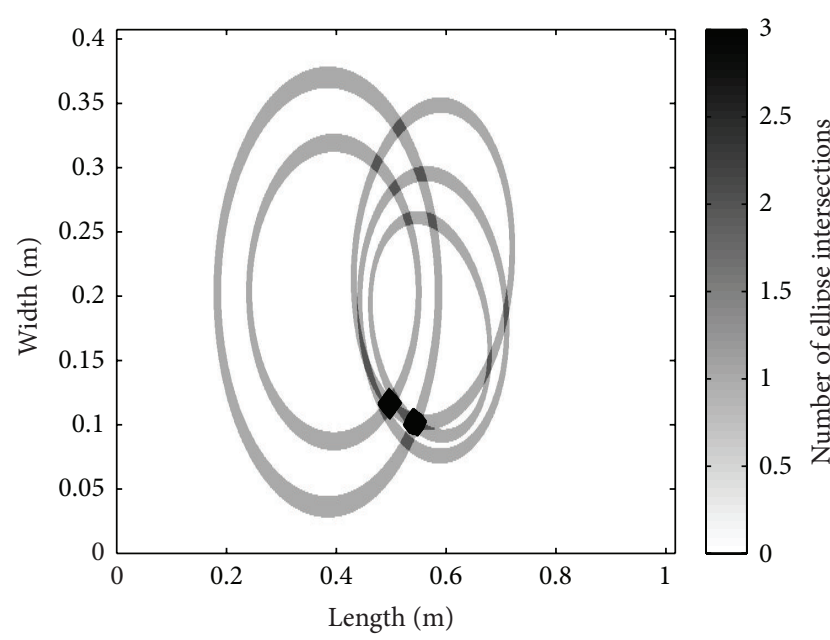

(a)

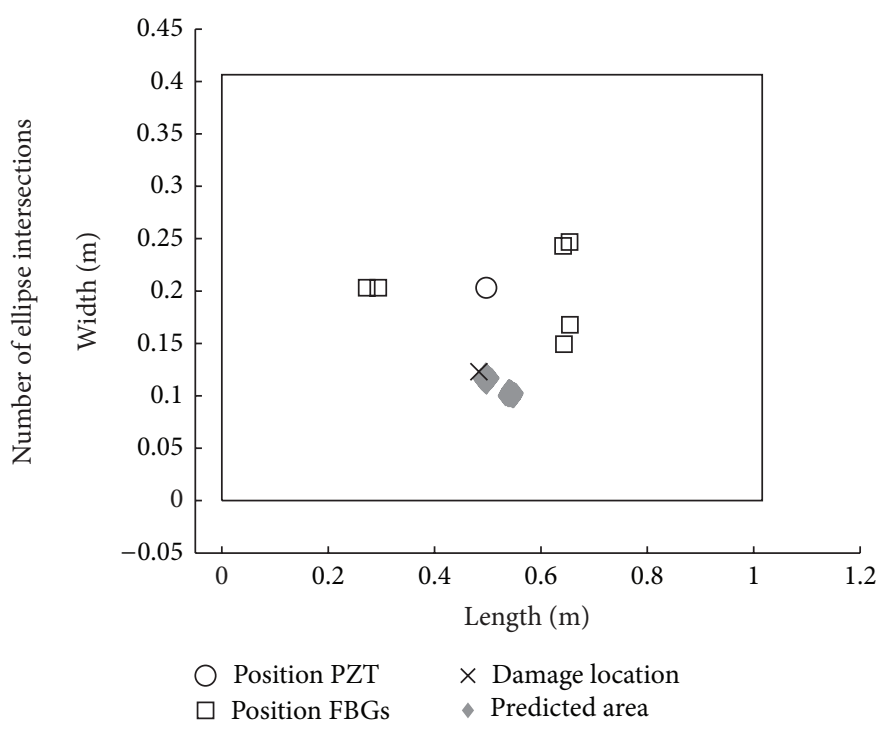

(b)

FIGURE 8: (a) Contour plot for the constructed ellipses and (b) comparison between the predicted damage and actual damage location, for a through the thickness damage.

means that the extreme locations obtained formed the outer boundary of the area which could be inspected. Connecting the extreme locations together formed the inspection area shown in Figure 7. The inspection area represents all the possible damage locations when signals up to $100 \mu$ s duration were analyzed; this area equaled $20.8 \%$ of the total area of the composite plate.

The developed algorithm was considered simple as it only used standard signal processing techniques and a localization technique, which was based on elliptical triangulation. The effectiveness of the algorithm was tested using realistic simulated damage, both in type and dimension. First, a $2 \mathrm{~mm}$ diameter through thickness hole was evaluated, followed by a bore hole $2 \mathrm{~mm}$ diameter with a depth of $0.65 \mathrm{~mm}$. After each introduced damage, the mentioned scan, data acquisition, and postprocessing were performed.

3.2. Through Thickness Hole. A $2 \mathrm{~mm}$ through thickness hole was introduced in the plate outside of the area bounded by the transducers; the constructed ellipses and the comparison between the predicted damage and actual damage location for the introduced damage are shown in Figure 8. The area between the two ellipses from each piezoceramic transducer/FBG pair, the ring, was assigned a magnitude of one, and other areas were set to zero. When all the rings were plotted, the highest number of ring intersections was stored 


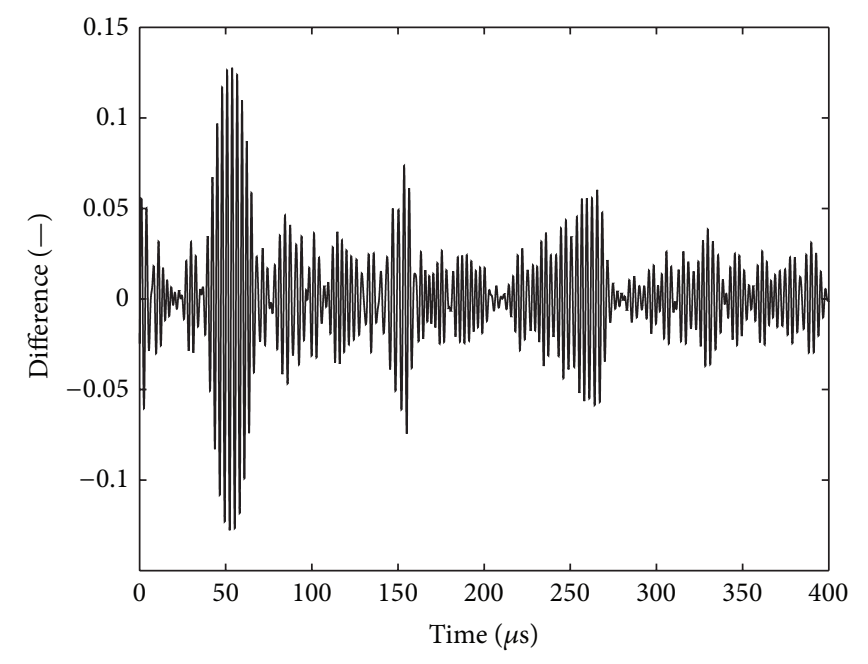

(a)

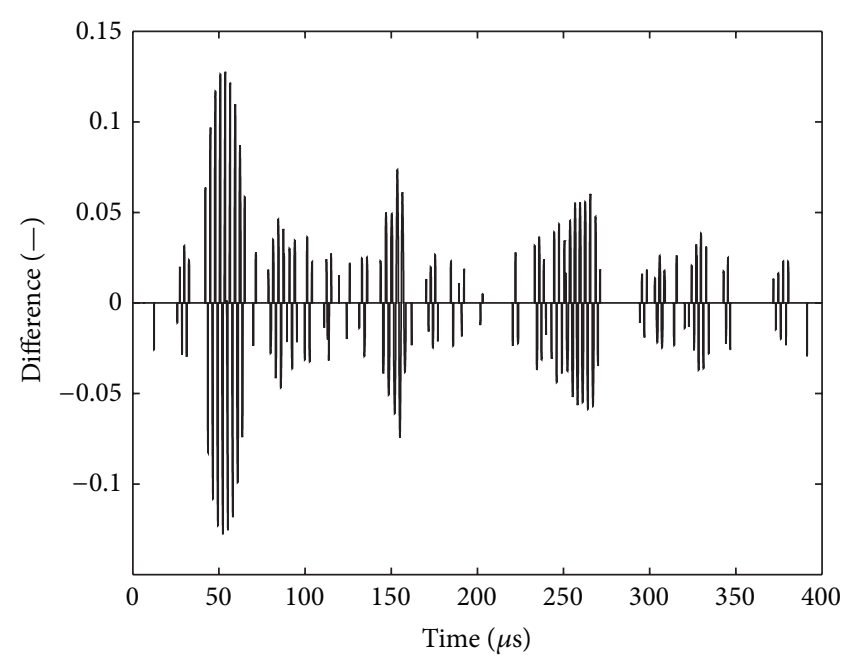

(b)

FIGURE 9: (a) Difference in signal of FBG 3 between baseline and damaged signal and (b) difference in signal of FBG 3 after the signal-band filter was applied.

and compared with the actual damage location to verify the damage detection and localization algorithm. In Figure 8(b) the location of the actual damage can be seen, marked with a cross, which is near the predicted damage area obtained by the algorithm. A closer examination of the area revealed that the actual damage was located $5 \mathrm{~mm}$ to the left of the predicted damage area.

The ellipses were constructed using two fixed velocities; however, better results could be obtained if the actual velocity in the FBG direction was used instead of a fixed range. The damage localization algorithm was based on the time of arrival of the largest echo only; an updated algorithm should be designed such that it can take the time of arrival of all the echoes into account to obtain a better prediction of the damage area.

3.3. Surface Damage: Bore Hole. The damaged state signalband for the $2 \mathrm{~mm}$ hole was then used as baseline for the second type of introduced damage. The second type of damage was introduced in the plate to mimic a surface damage; this damage was located on the opposite side of the plate with respect to the surface where the sensors were bonded. This simulated a realistic situation which could occur on an aircraft, where the damage would be located on the outer side of the skin, while the sensors would be bonded on the inner side, protected from the external environment. The surface damage was simulated by a $2 \mathrm{~mm}$ bore hole with a depth of $0.65 \mathrm{~mm}$. The same experimental procedure as for the first damage was repeated, and the obtained signals for the case of a surface damage are shown in Figure 9. The corresponding contour plot and localization image are shown in Figure 10. The estimated damage location was $25 \mathrm{~mm}$ away from the actual damage location. In this case, multiple peaks obtained from the comparison of the baseline and damage state corresponding signal-bands were considered and not just the highest peak as previously.

\section{Conclusions}

The proposed FBG interrogation architecture was assessed for the detection of Lamb waves excited by a piezoceramic transducer, in a representative CFRP aircraft skin. The capabilities and applicability of the system to SHM of aircraft were investigated. To detect damage, two signal-bands were required, one baseline signal-band and one damaged state signal-band. Signal-bands were considered to reduce the effects of noise in the transducer signals, improving considerably SNR and enabling repeatedly detection of the small damage reflection waves amplitudes in corresponding signals. The two signal-bands are compared to retrieve the outliers; a signal containing the echoes generated by the damage remained. The echo signal was then used to localize the damage in the composite plate. The damage localization algorithm was based on the time of arrival of the largest echoes. The time of arrival was multiplied by two fixed velocities, which represented the range of the $S_{0}$ wave velocity. The calculated distances were then used to construct ellipses for which the piezoceramic transducer and FBG were the focal points. The location at which most ellipses intersected was used to predict the region where the damage was located.

The algorithm was tested by evaluating two different types of damage: (i) a through thickness hole of $2 \mathrm{~mm}$ in diameter and (ii) a surface damage consisting of a $2 \mathrm{~mm}$ diameter bore hole with depth of $0.65 \mathrm{~mm}$ located on the backside of the plate with respect to the surface where transducers were installed. The damage detection and localization algorithm produced an image which indicated the predicted damage region, which was compared to the actual location of the damage. For the through thickness hole, the predicted damage region was $5 \mathrm{~mm}$ to left of the actual damage location. For the surface damage, the actual damage location was located $25 \mathrm{~mm}$ from the predicted damage region. The proposed hybrid SHM system was able to detect the introduced damage 


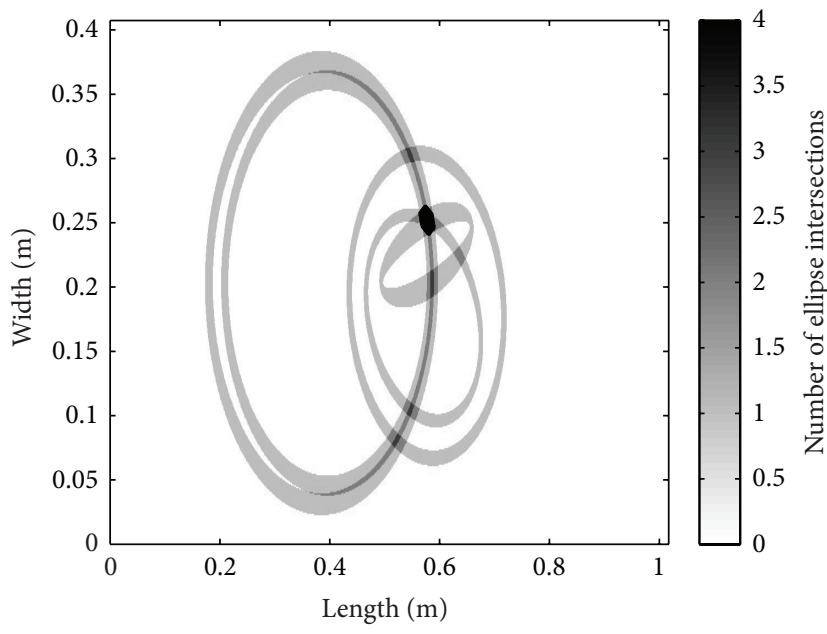

(a)

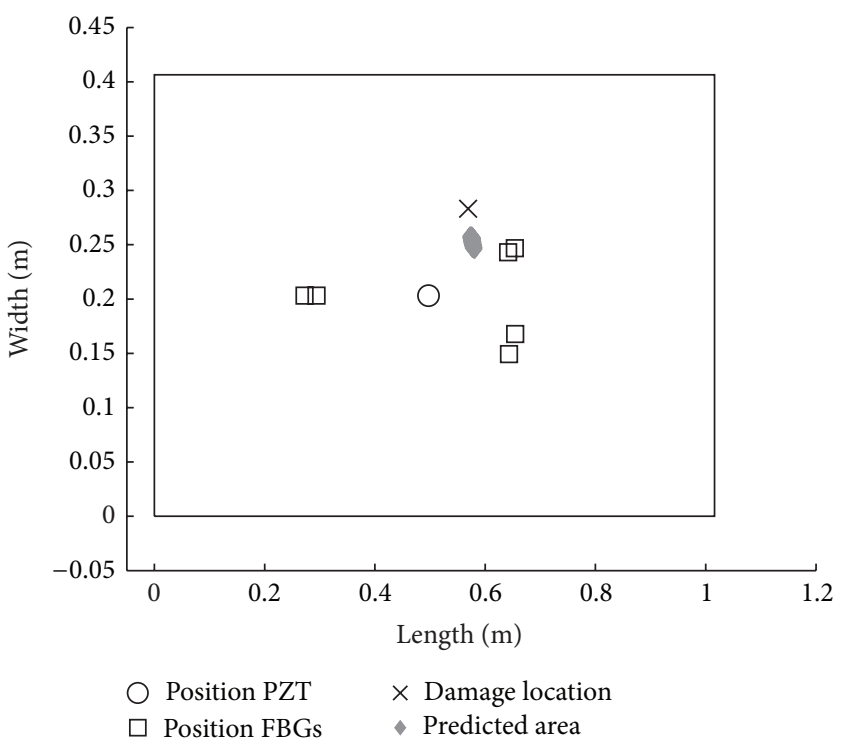

(b)

FIGURE 10: (a) Contour plot for the constructed ellipses and (b) comparison between the predicted damage and actual damage location, for a surface damage located on the back side of the plate.

and predicted the damage region with reasonable levels of accuracy. In real life applications, such a detection and localization capability would be useful to reduce overall NDE efforts and focus NDE techniques to a smaller region and therefore reduce time of inspection and maintenance, effort, and cost.

\section{Conflict of Interests}

The authors declare that there is no conflict of interests regarding the publication of this paper.

\section{Acknowledgments}

The support of the National Research Council Canada (NRC) on this research is gratefully acknowledged. The authors would like to kindly thank Dr. Honglei Guo, Dr. George Xiao, and Dr. Nezih Mrad for the use of their hardware and laboratory equipment necessary for this research project. The authors would also like to thank the Faculty of Aerospace Engineering at Delft University of Technology (TU Delft) for their financial support. Furthermore, the authors would like to express their gratitude to Professor Dr. Ir. Rinze Benedictus and Ir. Jan Hol for their technical guidance.

\section{References}

[1] R. Baldev, T. Jayakumar, and M. Thavasimuthu, Practical NonDestructive Testing, Woodhead Publishing, 2nd edition, 2002.

[2] W. Statszewski, C. Boller, and G. Tomlinson, "Health monitoring of aerospace structures: smart sensor technology and signal processing," Expert Systems with Applications, vol. 39, no. 9, 2004.

[3] "Guidelines for implementation of structural health monitoring on fixed wing aircraft," Tech. Rep. SAE ARP6461, 2013, http://www.sae.org/.
[4] D. C. Betz, G. Thursby, B. Culshaw, and W. J. Staszewski, "Structural damage location with fiber Bragg grating rosettes and Lamb waves," Structural Health Monitoring, vol. 6, no. 4, pp. 299-308, 2007.

[5] Z. Su and L. Ye, Identification of Damage Using Lamb Waves: From Fundamentals to Applications, vol. 48 of Lecture Notes in Applied and Computational Mechanics, 2009.

[6] F.-K. Chang, J. F. C. Markmiller, J.-B. Ihn, and K. Y. Cheng, "A potential link from damage diagnostics to health prognostics of composites through built-in sensors," Journal of Vibration and Acoustics, vol. 129, no. 6, pp. 718-729, 2007.

[7] L. Qiu, M. Liu, X. Qing, and S. Yuan, "A quantitative multidamage monitoring method for large-scale complex composite," Structural Health Monitoring, vol. 12, no. 3, pp. 183-196, 2013.

[8] H. Lamb, "On waves in an elastic plate," Proceedings of the Royal Society of London, Series A, vol. 93, no. 648, pp. 114-128, 1917.

[9] G. Keiser, Optical Fiber Communications, McGraw-Hill, New York, NY, USA, 4th edition, 2011.

[10] T. H. Loutas, A. Panopoulou, D. Roulias, and V. Kostopoulos, "Intelligent health monitoring of aerospace composite structures based on dynamic strain measurements," Expert Systems with Applications, vol. 39, no. 9, pp. 8412-8422, 2012.

[11] D.-H. Kim, B.-Y. Koo, C.-G. Kim, and C.-S. Hong, "Damage detection of composite structures using a stabilized extrinsic Fabry-Perot interferometric sensor system," Smart Materials and Structures, vol. 13, no. 3, pp. 593-598, 2004.

[12] A. Raghavan and C. E. S. Cesnik, "Review of guided-wave structural health monitoring," Shock and Vibration Digest, vol. 39, no. 2, pp. 91-114, 2007.

[13] L. Qiu, S. Yuan, X. Shi, and T. Huang, "Design of piezoelectric transducer layer with electromagnetic shielding and high connection reliability," Smart Materials and Structures, vol. 21, no. 7, Article ID 075032, 2012.

[14] N. Takeda, Y. Okabe, J. Kuwahara, S. Kojima, and T. Ogisu, "Development of smart composite structures with smalldiameter fiber Bragg grating sensors for damage detection: 
quantitative evaluation of delamination length in CFRP laminates using Lamb wave sensing," Composites Science and Technology, vol. 65, no. 15-16, pp. 2575-2587, 2005.

[15] H. Tsuda, "Ultrasound and damage detection in CFRP using fiber Bragg grating sensors," Composites Science and Technology, vol. 66, no. 5, pp. 676-683, 2006.

[16] P.-M. Lam, K.-T. Lau, H.-Y. Ling, Z. Su, and H.-Y. Tam, "Acousto-ultrasonic sensing for delaminated GFRP composites using an embedded FBG sensor," Optics and Lasers in Engineering, vol. 47, no. 10, pp. 1049-1055, 2009.

[17] Z. Wu, X. P. Qing, and F.-K. Chang, "Damage detection for composite laminate plates with a distributed hybrid PZT/FBG sensor network," Journal of Intelligent Material Systems and Structures, vol. 20, no. 9, pp. 1069-1077, 2009.

[18] Y. Tan, L. Cai, B. Peng, and L. Meng, "An investigation of structural damage location based on ultrasonic excitation-fiber bragg grating detection," Advances in Acoustics and Vibration, vol. 2013, Article ID 525603, 6 pages, 2013.

[19] B. Rocha, Structural Health Monitoring of Aircraft Structures, Universidade Técnica de Lisboa, Instituto Superior Técnico, 2011.

[20] B. Rocha, D. Barazanchy, R. Sevenois, H. Guo, G. Xiao, and N. Mrad, "Assessment of fibre optic sensor architectures for structural health monitoring," in Proceedings of the 9th International Workshop on Structural Health Monitoring (IWSHM '13), pp. 470-477, Palo Alto, Calif, USA, 2013.

[21] C. Kassapoglou, Design and Analysis of Composite Structures with Applications to Aerospace Structures, John Wiley \& Sons, 1st edition, 2010.

[22] B. Rocha, M. Yanishevsky, A. Beltempo, R. Rutledge, N. Bellinger, and M. Martinez, "Global framework for the assessment, development and demonstration of structural health and load monitoring systems," in Proceedings of the Canadian Aeronautics and Sp ace Institute (CASI) 60th AERO Conference, Toronto, Canada, 2013.

[23] C. Davis, S. Tejedor, I. Grabovac, J. Kopczyk, and T. Nuyens, "High-strain fiber bragg gratings for structural fatigue testing of military aircraft," Photonic Sensors, vol. 2, no. 3, pp. 215-224, 2012. 

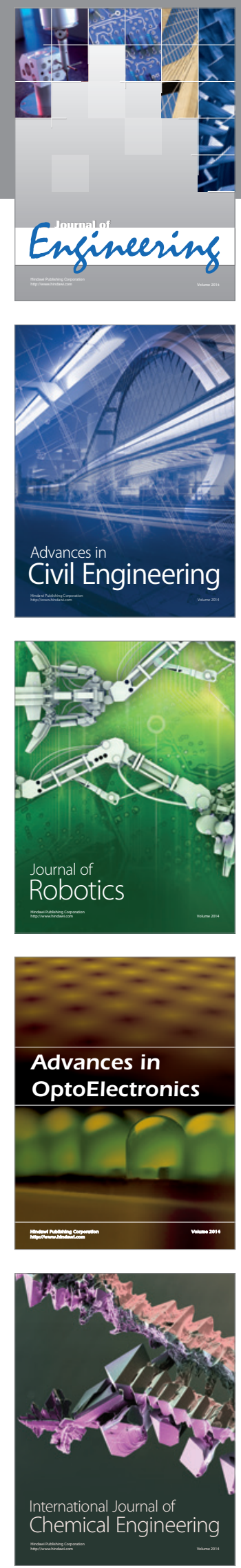

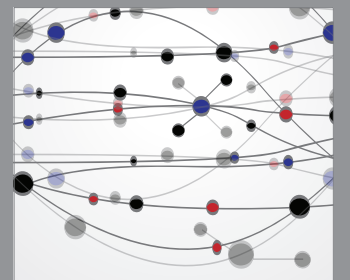

The Scientific World Journal
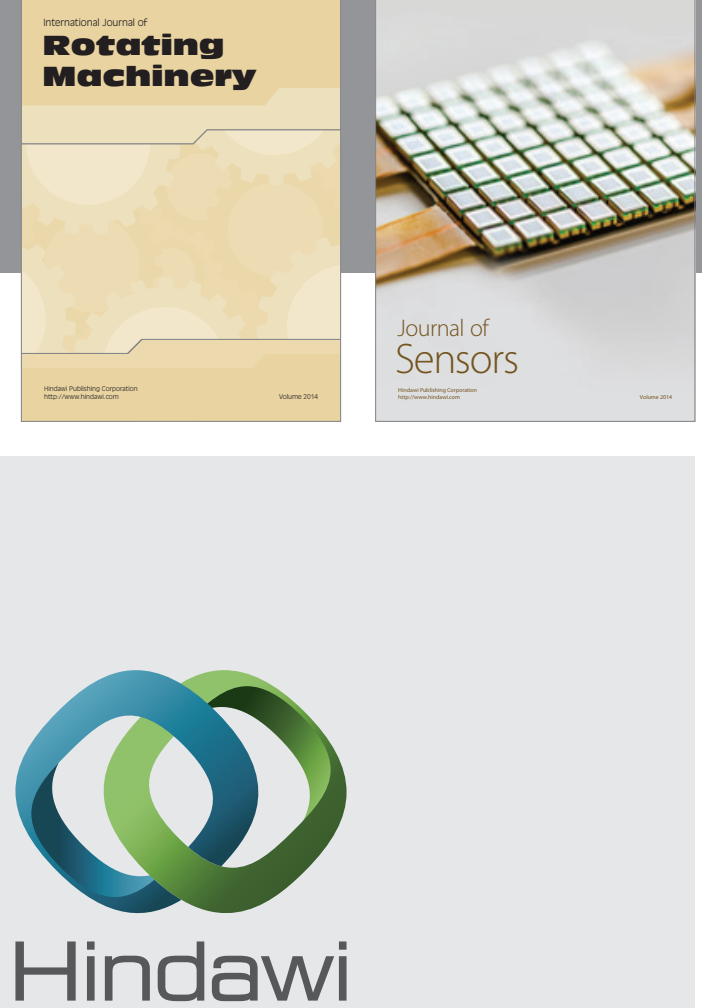

Submit your manuscripts at http://www.hindawi.com
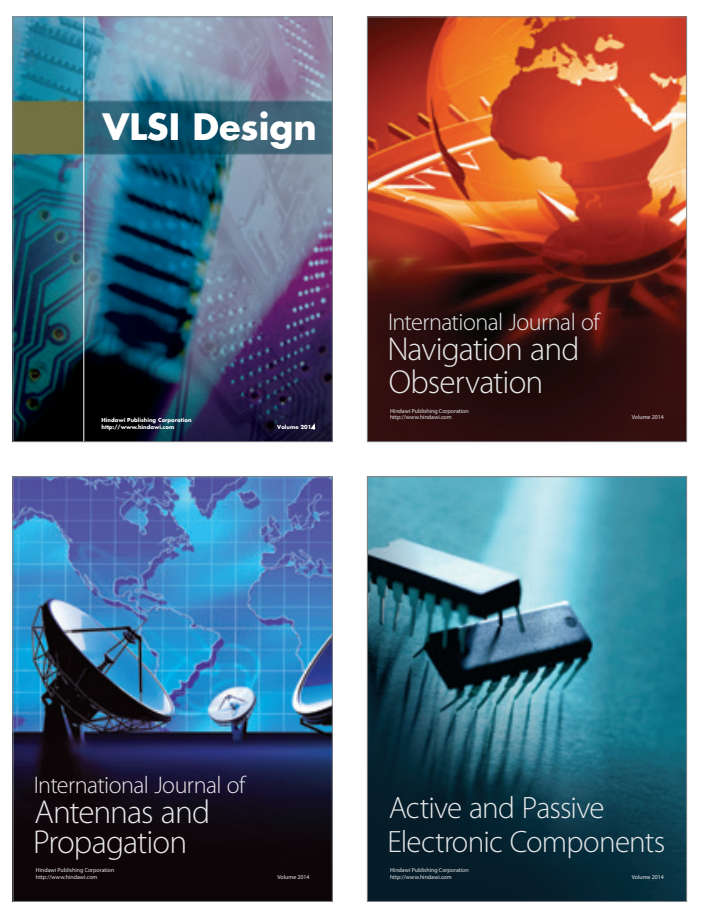
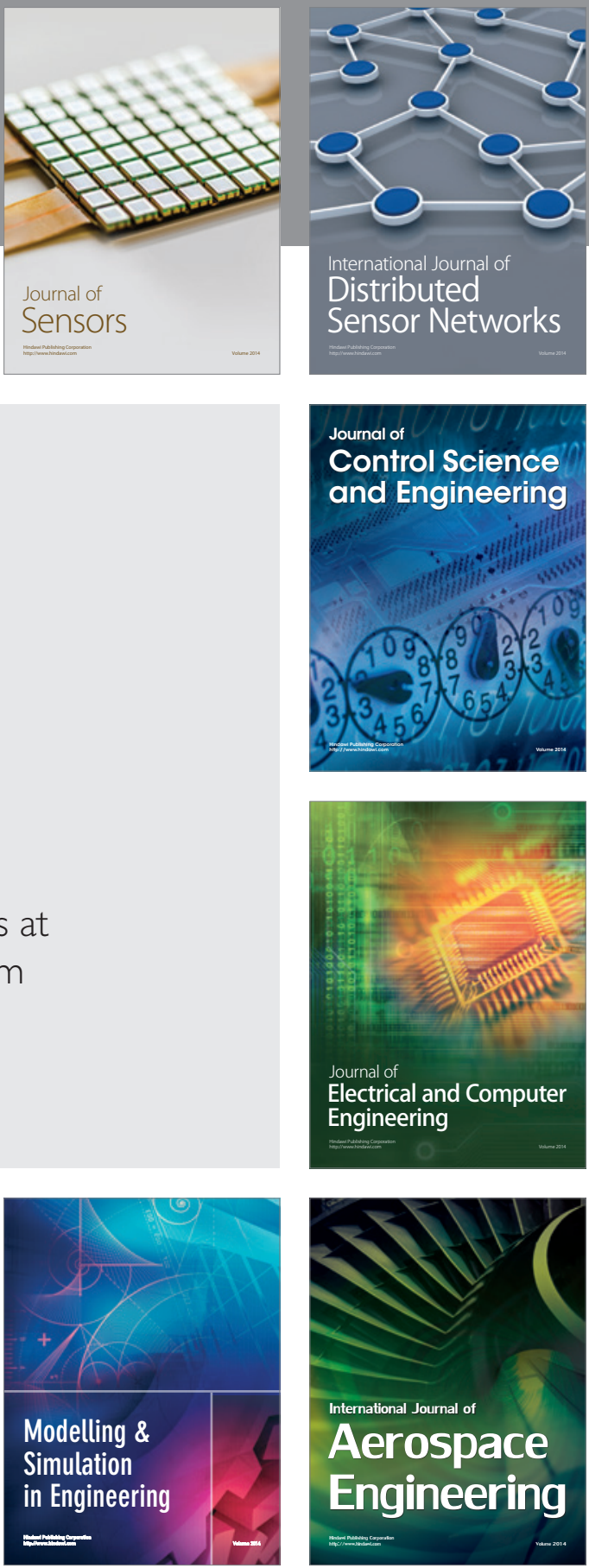

Journal of

Control Science

and Engineering
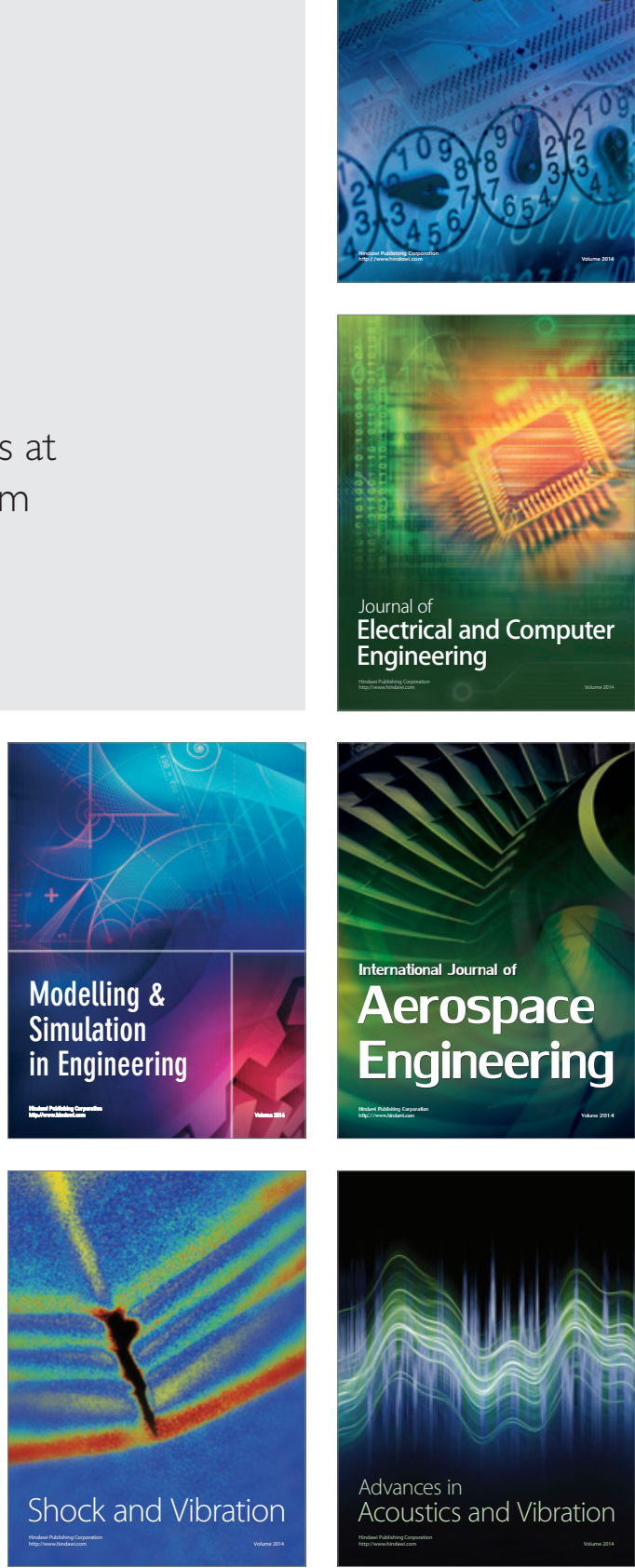